therefore, matter is prominent ; in biological forms the oncrgy-aspect is apparently more active and life is more evident; in the human kingdom the solective power functions through a wider gamut than the instinctive intelligence of animals, and mind predominates.

As this polar function does not refer to 'size', its matter-aspect must be of a different tenuity than ' matter' so called, and hence imperceptible to us before it is precipitated or condensed into concrete states.

The supposition of an evolution through these grades is as fantastic in the present state of scientific knowledge as the ancient Hermotic axiom, "The stone becomes a plant, the plant an animal, the animal a man, and man a god". Such a procoss implies interacting cycles of evolution producing modifications of the inner 'structures' of bodies in the successive grades. The conception of the ancients was that evolution is a progressive functioning of the mysterious polar principle actively synthesising more and doeper fields within a single organism.

W. W. L.

ThF presidential address of Dr. H. H. Dale on "The Biological Nature of the Viruses", in NATUre of Oet. 10, puts clearly one aspect (the pathological one) of substances that may possibly lead to the most notable juncture in contemporary thought. General Smuts-and he is but one of several-adumbrates, in his holism, a synthesis of mental with physical science. The viruses, including as they do the minutest particles to which life is attributed, give promise of leading to such a synthesis. Some appear to be so small that they raise the question whether, in their series, life does not exist in sizes below any protein molecule. This class of questions is at present engaging much attention in Amorica and Germany, and some investigators postulate the hydrogen atom as perhaps a member at the lower end of the series of living things. Dr. Dale, of course, does not consider this point. If such a view should obtain general acceptance, the notable juncture would occur that the two greatest lines of modern thought-the evolutionary biology of Darwin and the physics of Newton and Faradaywould find a common meeting point in the atom.

May I presume to mention that $\mathrm{I}^{1}$ and others have tried to show that directivity and affective fecling are inherent in the processes of evolution. If so, it is fair hypothosis to attribute these to the atom, if ever it is adopted as a member of the series of living things. Dr. Dale, in his acutely logical treatment of the new facts, makes some sound remarks, one of which is: "Let us recognize that the evidence is not perfect, but beware of a merely sterilizing scepticism". (F. d'Herelle, "The Bacteriophage"; Med. Rescarch Council (Gt. B.), "The Viruses"; E. W. Schultz, "The Ultrascopic Viruses", in Scientific Monthly, Nov. 1930; H. Busher, "Where Life Begins ", Atlantic Monthly, July 1930 ; and authorities cited.)

W. D. LighthaLL.

McGill University, Montreal, Oct. 27.

1 "The Person of Evolution" (Macmillan, 1930), Chaps. iv. and v.

\section{A Two-Dimensional Space Lattice?}

ON cooling a warm aqueous solution of dimethylthallium iodide, or on adding a ten per cent solution of potassium iodide, preferably slightly alkaline, to its cold saturated aqueous solution, part of the thallium compound separates on the surface in a regular pattern. Each crystal is equidistant from six others. 'The size of the pattern varies, being smaller the more rapidly crystallisation is allowed to take place. The effect is not permanent; after about half an hour the arrangemont breaks down and the crystals group together in irregular aggregatos, which collect at the sides. In a slightly acid solution the regular

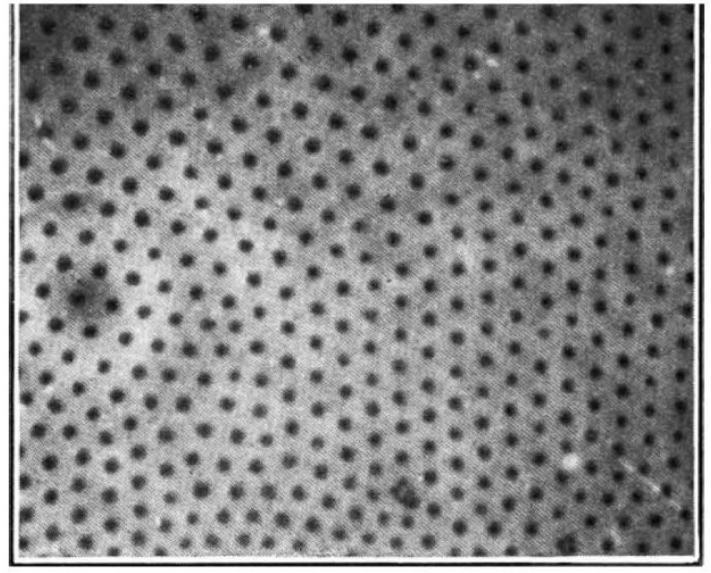

FI*. 1.

arrangement breaks down in a few seconds. I should be glad to know if other similar effects are known, and also to be given an explanation which I am unablo to supply.

Fig. 1 is an enlargement of part of a photomicrograph, kindly taken by Dr. W. J. Elford by focusing on to the surface of a small quantity of the solution allowed to crystallise on a microscope slide.

Chemical Department,

R. C. Menzies.

The University, Bristol, Oct. 13.

\section{Goagulation of Golloids by Electrolytes.}

Aтtночgr the impuritios present in a colloid considerably modify its behaviour as regards coagulation by electrolytes, the importance of this point is still not sufficiently realised by many workers in colloid chemistry. It has been pointed out by one of us (Desai) ${ }^{2}$ that a colloid can be made to show either normal or abnormal behaviour towards the dilution rule, namely, that the greater the concentration of a colloid, the greater the amount of an electrolyte required to coagulate it, by varying the amount of the poptising agent present in the colloid by subjecting it to dialysis.

In a recent note in NATURE we (Nabar and Desai) ${ }^{2}$ have shown that in the case of colloidal gold the stability as defined by the floceulation value with $\mathrm{KCl}$ goes hand in hand with the charge on the colloid when it is subjected to dialysis. Since then we have made measurements of the charge on colloidal ferric hydroxide dialysed and diluted to different exterits in the prosonce and absence of added electrolytes. The following is a summary of the results :

1. On the addition of increasingly small amounts of $\mathrm{KCl}$ and $\mathrm{MgCl}_{2}$ the charge first increases and then decreases, the initial increase being greater with the concentrated sol than with the dilute one.

2. The initial increase in the charge is not noticeable with $\mathrm{K}_{2} \mathrm{SO}_{4}$, even when it is added in very small amounts.

3. With the progress of dialysis, although the charge

No. 3239, VoL. 128] 